\title{
Rethinking Level 5: Distributed Cognition and Information Fusion
}

\author{
Maria Nilsson \\ School of Humanities and Informatics \\ University of Skovde \\ Sweden \\ maria.nilsson@his.se
}

\author{
Tom Ziemke \\ School of Humanities and Informatics \\ University of Skovde \\ Sweden \\ tom.ziemke@his.se
}

\begin{abstract}
The focus of most information fusion research, so far, has been on the technology, i.e. information processing in machines. However, the importance of also understanding human information processing, and the interaction between humans and machines, is gaining increasing recognition. This position paper argues that a distributed cognition perspective, which considers cognitive processes not as taking place solely inside people's heads, but as distributed over human thought processes and the material, social and organisational environment they are embedded in, may help to provide a deeper understanding of information fusion processes.
\end{abstract}

Keywords: Information Fusion, JDL Model, Distributed Cognition, Human-Computer Interaction, Context

\section{Introduction}

Information fusion is becoming increasingly important in the light of current developments of complex information technologies. Information fusion can be found in many different areas including, but not limited to, military applications, bioinformatics, manufacturing, agriculture, crisis management, etc. [1-4]. The most commonly used model to describe levels and elements of information fusion is currently the JDL model [1]. It explains, from a functional point of view, the hierarchical process of information fusion where typically input data from multiple sensors is transformed/condensed bottom-up to, for example, graphical displays of the most relevant information for human decision-makers. Most research in information fusion has its focus on the technical aspects such as probability and statistics, decision and estimation theory, pattern and image recognition, approximate reasoning techniques such as fuzzy logics, and other artificial intelligence tools [4], i.e. the techniques and methods used at levels 1-3 of the JDL model. Cognitive science, on the other hand, has so far had a relatively limited impact, partly in the form of 'cognitive modelling', i.e. the use of human-like or human-inspired techniques at levels 1-3 [5-7], and partly in the form of studies of human computer interaction (HCI), sometimes referred to as a "level 5" [1,8]. This position paper will focus on the latter.

Several researchers have acknowledged that there is a lack of research identifying the various HCI and cognitive issues [5, 8-10]. For instance, the need for adaptive interfaces to encourage human centred fusion has been recognised [8]. Also, social expectations have been identified as an area which needs to be investigated further [9]. In fact, it has been argued that the effectiveness of the overall information fusion process is affected by the utilisation of HCI [1]. In 2005, there was an editorial of the Information Fusion Journal dedicated to HCI issues [10].

Although there is, as briefly exemplified above, a growing understanding of the crucial role of the human user in information fusion, in our opinion, the perspective needs to be extended from thinking of information fusion as a process solely taking place in computer systems, to thinking of information fusion processes as processes including humans and their surroundings. That means, we need to widen the perspective of the fusion process from information fusion systems to information fusion processes (cf. Figure 1). An information fusion system often refers to the type of computer system characterised by the JDL model (levels 0-3), the process of transforming low-level sensory data to higher-level more abstract information. An information fusion process, on the other hand, also includes the context of the user(s), the material surroundings, the organisation they are part of, etc.

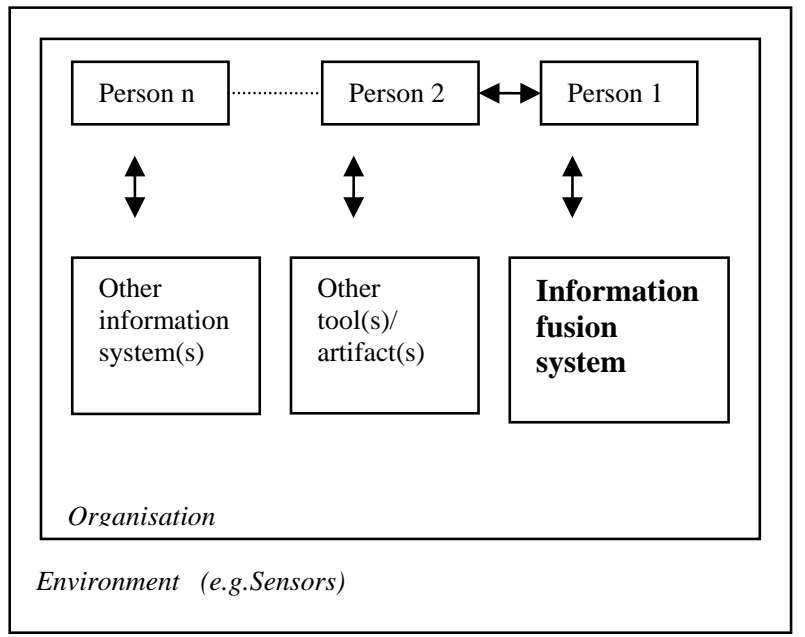

Figure 1: An information fusion process (an information fusion system integrating with its surroundings) 
The need to pay more attention to the surrounding environment has also been pointed out by Brehmer [12] who argued that actions always have an effect on the environment which needs to be accounted for. In a similar vein, Blasch and Plano [30] emphasised the importance of context for user refinements to aid the fusion process, and Hall and McMullen [4] argued: "By rethinking the HCI for data fusion, we may be able to re-engage the human in the data fusion process and leverage our evolutionary heritage” (p. 336).

A related shift has occurred in cognitive science and artificial intelligence (AI) research in the last 15-20 years. While traditionally cognitive science and AI focused much on computational thought processes inside the heads of individuals, more recent work on distributed/situated/embodied cognition views cognition not as taking place solely inside people's heads, but as distributed over human thought processes and the material, social and organisational environment they are embedded in. In this position paper we argue that taking a distributed cognition perspective in information fusion will be useful in achieving a deeper understanding of information fusion processes i.e. the embedding of information fusion systems in their cognitive and organisational context.

\section{The JDL model}

The commonly used JDL model is a functional model which describes the hierarchical process of information fusion from low-level sensory data to more abstract, higher-level information. There have been various extensions to the JDL model throughout the years [1]. The most commonly used model includes level 0 to level 4 [13]. However, there is a discussion whether or not level 4 should really be considered a 'level' in the JDL model $[13,1]$. It has been argued that the so-called level 4 is actually a meta-process, i.e. a process which controls and optimizes the rest of the system and, thus, not a functional level itself (however, the details of this discussion are beyond the scope of this article). As briefly mentioned above, cognitive aspects have been incorporated to the JDL model both in levels 1-3 (e.g. cognitive modelling) and the higher levels (e.g. a suggested "level 5"), discussed in more detail below.

\section{$2.1 \quad$ Level 5}

A “level 5" was first suggested in 2000 by Hall, Hall and Tate [14] in order to explicitly account for functions associated with HCI. They argued that we need to remove the "HCI bottleneck" [14], which refers to the fact that broadband sensor data is fused through a narrow channel, i.e. the computer screen which the user interacts with, to be analysed by a broadband human being. It can be argued that the HCI interface becomes a bottleneck which prohibits humans from using their extensive pattern recognition and analytical skills to infer the information presented. Level 5 typically includes both cognitive and HCI issues, but is commonly referred to as "Level 5: cognitive refinements" $[8,14]$. The level includes specific technical aspects which can be used for achieving cognitive refinements and recommended research areas, such as:

- time compression/expansion: human senses are especially optimised towards change detection; development of time compression and time expansion replay techniques could assist the understanding of an evolving tactical situation

- negative reasoning enhancement: humans have a tendency to ignore negative information and only seek information that confirms a hypothesis; techniques which exploit the idea of "absence of evidence" and "counter-evidence" should be developed

- pattern morphing methods: methods should be developed to translate patterns of data into forms that are more optimised for the users' interpretations

- uncertainty representation: visual, auditory and haptic techniques should be developed in order to improve the detection of uncertainty

Blasch and Plano [11] suggested re-labelling "level 5: cognitive refinements" as "level 5: user refinements" and introduced the JDL-User model. The goal of the modified level is to extend the human capabilities. Their definition of level 5 is as follows [11]: “adaptive determination of who quires information and who has access to information (e.g. information operations) and adaptive data retrieved and displayed to support cognitive decision making and actions (e.g. altering the sensor display)" (p. 273). The aim with the level 5 is to support: cognitive workload, trust, attention, and situation awareness (see also [29] for a more detailed discussion of user refinements). However, the above definition has more recently been suggested to include the ending: "... support cognitive decision making and actions (e.g. altering the sensor display) given social and political contexts" [30] (p.195). This definition acknowledges the importance of the social and political context for the usage of information fusion systems. However, it should also be noted that incorporation of a level 5 into the JDL model has not yet achieved common usage or acceptance by the fusion community [13].

\section{Alternative models}

The JDL model is not the only model used to capture and characterize information fusion systems. There are models which have tried, to some degree, to incorporate the human decision maker. For instance, Kokar et al. [9] approached information fusion from a design process perspective. They proposed a new object oriented reference model for data fusion systems where the data fusion process is top-down (the system constraints are considered first). Within this model the human capabilities can be easily accommodated, since no distinction is drawn between human and computer objects until the model is viewed from the realization perspective.

Another model is the Omnibus process model [15] which aims to capture the information fusion process by 
using a general terminology. The model includes a process flow chart, a dual perspective prescription for using it and a structured repository of fusion knowledge. Moreover, feedback loops are explicitly acknowledged in the model.

There are also models which have focused on the human decision process. Most prominently, Boyd's OODA (Observe-Orient-Decide-Act) loop [16] originated from the military domain. It was intended to capture the command and control process and is still today the most commonly referenced model. It consists of four activities, i.e. observe- observe the environment, orient- position yourself in the environment, decide- make a decision of what to do next, act- perform the decision. The aim of the model is to enable faster decisions by identifying both your own decision steps and your opponent's. However, it has been argued that the model gives a static picture of decision making, and therefore the dynamic OODA (DOODA) loop has been developed as an attempt to include the dynamic nature of the command and control process [12].

The DOODA loop model [12] was suggested in a military setting in order to capture the actual decision process by combining ideas from the OODA loop and cybernetic models. The model is divided into different functions (information collection, command concept, sense-making, planning, decision/order, military activity, effect). Some characteristic functions are as follow:

- Command concept - an overall concept of how an operation is to be conducted. This is one of the most important concepts in the model.

- Sensemaking - here, "sensemaking" refers to an understanding of the current situation and what can be done, the result of this function is a collective activity of the commander and his staff aimed at an action. However, it was emphasised that "sensemaking" should not be confused with the concepts of situation awareness. Nosek [17] also used the concept of "sense making” when describing how knowledge emerges in teams which interact with complex system. For instance, according to this view, the team creates the future by interacting with objects and the surrounding environment.

- Effect - the decision has an explicit, notable effect in the surrounding environment.

- Arrows- arrows determine the flow and feedback loops between the different functions, although overlaps between the different functions are acknowledged.

The goal of the model is to identify functions for an effective command and control process. The model may appear complex, however, the nature of a decision is complex; hence, a complex model cannot be avoided according to Brehmer [12].

To summarize, the studies briefly discussed above indicate that humans should and can successfully be incorporated into the information fusion process. As discussed in the following section, we propose a distributed cognition perspective as a valuable complement to capture the actual information fusion process.

\section{Distributed cognition}

\subsection{A cognitive science perspective}

The traditional (mainstream) view in cognitive science focused on mental processes inside the heads of individuals. However, more recently distributed cognition [18] has become a well established perspective in cognitive science, focusing not only on a single individual's internal processes, but also on the interactions with the surroundings such as other individuals (groups), artefacts and other types of (information) systems as part of cognitive processes. In other words, cognition, is not longer explained in terms of information processing at the level of the individual, instead, cognition is argued to be better explained as a distributed phenomena [19]. Hence, cognition can be considered as distributed in a threefold sense:

- distributed across individuals in a group or organisation

- distributed between human-internal (e.g. memory) and -external mechanisms (e.g. computer systems, material and/or social environment)

- distributed over time

A key aspect of distributed cognition [18] is that there are individuals with usually well defined roles and tasks who interact with each other and with computer systems, e.g. information fusion systems; everyone possess their own expert knowledge but something more, something new emerges in the interaction between them, e.g. an information fusion process. In other words, one might say that the sum of the parts is greater than the whole. Thus, in a distributed cognition perspective the approach is that the cognitive process is not only within the heads of individuals; rather, the cognitive process is created in the interaction with the computer system and other individuals.

As a researcher one would be interested in the interdependencies between the individuals and the information fusion systems i.e. "in distributed cognition, one expects to find a system that can dynamically configure itself to bring subsystems into coordination to accomplish various functions. A cognitive process is delimited by the functional relationships among the elements that participate in it, rather than by the spatial collocation of the element" [19] (p. 176).

Approaching problems with a distributed cognition perspective could give the researcher or system developer a new way to understand problems. Indeed, in distributed cognition, the analysis (typically using in-depth ethnographic studies) deals with representing raw data collected at different levels of abstraction and detail, with a focus on changes in representation states [31]. Here, representational states refer to how knowledge and information are transformed to conduct an activity/action, e.g. the information displayed by a computer system transforms its state in response to a user keying in a command. For example, consider data from an ethnographic study of the activity of flying to a higher altitude in a plane, the coordinated activity of an air traffic 
controller, a co-pilot, pilot and the plane itself (i.e. a computer system) [31]. In this example, the distributed cognition approach/analysis identified [31]:

- the distributed problem-solving that took place

- the role of verbal and non-verbal behaviours

- the various coordinating mechanisms that were used

- the different ways of communication that took place as the collaborative activity progressed

- how knowledge was shared and accessed

To give another example, in distance education one could have one or more students which collaborate through, for example, the internet. Video conferences are a common tool to use for interacting between the teacher and the students. In this case, Schrire [20] identified, using a distributed cognition perspective, the usage of the conferences and the interactions between the individuals. In particular, the author explained the emerging higherorder thinking which were characteristic to the conferences studied (which would have been difficult to identify without a distributed cognition perspective).

Furthermore, distributed cognition has been used to identify breakdowns in design processes. Busby [21] studied errors in the design process of a chemical process plant and a power generating plant. The problem studied was of the type "a designer forgets to include a particular kind of feature to the design". The author concluded that "communication problems" were the most common cause. A typical communication problem could be a failure in the verification of one person's results by another. Using a distributed cognition perspective enabled Busby "to reveal this kind of nested structure, its dependences on the interference of the participants, and the root of these inferences in their historical experience of earlier episode of similar kinds” [21] (p. 253). One could argue that the model of the internal organisational structure could be hard to identify without a distributed cognition perspective.

Holland et al. recently [19] proposed that distributed cognition should be used as a new foundation for HCI since in today's society, one does not only work in front of a single computer, in fact, one most often work with connected ubiquitous computers. Thus, distributed cognition can be used to understand the emerging dynamic interactions which take place in our networked world of information and computer mediated interactions, i.e. the characteristics of today's society [19]. The following subsection discusses in further detail how distributed cognition can be relevant to information fusion.

\subsection{Distributed cognition in information fusion}

In much information fusion research there has been a more or less implicit assumption that it is always better to fuse since adding more information can only help and not hinder the user when making interpretations [3]. However, as Dasarathy [4] points out, there are a number of studies which indicate that more information is not always better, i.e. depending on the presentation/usage of the information it may be more or less complicated to make a decision. For an information fusion system developer, this balance can be hard to recognise. Using a distributed cognition framework it might be possible to identify the interaction between the human and the information fusion system, as similar to [21, 31], and thereby, identify the breakdowns (and when to fuse). To our knowledge, in today's research in information fusion, the power of distributed cognition is not elaborated, even though, the subject is touched upon, as Blansch and Plano [32] acknowledge "A user fuses data and information over time and places and acts through their world mental model- whether it be in the head or with graphical displays, tools, and techniques” (p. 195). There are many different domains within information fusion which can benefit from a distributed cognition perspective due to the distributed nature of many fusion systems.

For example, the defence domain is, by far, the most developed area to exploit information fusion. The focus in the defence domain is predicted to be on creating the new "network-centric warfare". It is acknowledged that the network-centric environment demands distributed decision making (e.g. the decision is distributed between different individuals and locations). The aim of networkcentric warfare is to enhance military capacity through the power of information from the following assumptions [22]:

- Information is shared

- Situation awareness and commander's intent is available to all levels

- Operations are effects based and synchronised

- Decision-making should be collaborative

An important focus in network-centric warfare is to create shared situation awareness, "shared awareness aspires to achieve a common state of understanding within a group through the exchange of data and information" [22] (p. 4). To be able to create shared situation awareness it is important that the commander's intent is accessible and understandable, also, it is important to understand that shared situation awareness can only be developed over time. The concept of shared situation awareness might seem vague and as a researcher it can be difficult to identify the meaning of "shared situation awareness". Using a distributed cognition one has the terminology needed to identify the interactions between the different individuals and the interactions connected to the information fusion system, hence, the actual information fusion process can be captured.

The dynamic and distributed nature of the military command and control process is another related example where distributed cognition could make contributions. For instance, Bisantz at al. [23] developed a framework for aided, adversarial decision making (AADM), i.e. the use of decision aids based on information fusion in a cooperative and distributed defence domain. The authors identify a number of issues which needs to be considered when designing decision aids in the area of AADM. Trust is identified as a major issue defining the usage of the decision aid. It is important to know how much the user relies on the system and when that trust decrease due to the possible information attacks and failure in the 
automation of the system. Another issue is the distributed nature of AADM. Multiple locations, individuals and groups of individual together with the adversary force needs to be considered. Therefore, issues related to situation awareness are important for the interpretation of a decision. The authors also identified a number of specific dimensions to AADM (locus of attack, malfunction level, causes of failure or corruptions, time patterns of failure, predictable or unpredictable degradation).

A distributed cognition perspective on AADM would provide a common ground for the different factors presented and identify overlaps between different sources of data. Also, with a distributed cognition perspective one would more easily detect disruptions in the decision aid made by an adversary force. Indeed, a characteristic of AADM is the presence of the adversarial force and the vulnerability it involves e.g. information attacks.

Also, a distributed cognition perspective can, more specifically, be used when performing threat assessment/situation assessment. "Often the key indicators of potential, imminent or current threat situations are in the relationships among people and equipment that are not in themselves distinguishable from common, notthreatening entities" [28] (p. 2). For instance, [28] developed a new approach for threat assessment i.e. characterising, predicting and recognising threat situations. The goal was to establish a systematic approach to automating some of the above mentioned functions. Here, distributed cognition can be used as a valuable complement and tool in guiding the researcher in when to automate which functions.

Moreover, [11] proposed the usage of humans as to best locate and ID targets in group tracking, however, having a distributed cognition perspective would provide further understanding of the interaction between the human and the information fusion system, and thus, help to optimise the utilisation of the human capabilities. Not to forget, the value of context in information fusion can already be seen in, for instance [30]. Here, context (i.e. the social-political environment translated into mission goals) has been shown to be useful for target recognition. This further supports the notion that context is a valuable part of information fusion processes.

\section{Discussion}

As humans, we have extraordinary skills which help us interact with the surrounding environment. We are good at recognizing patterns, identifying geographic proximity, and looking for relationships between natural objects and man-made objects such as bridges, roads and buildings [8]. However, in information fusion one has yet to learn how to exploit this knowledge when designing complex computer systems. Some suggestions for future work and the added value a distributed cognition perspective provides are elaborated in the following subsections.

\subsection{Humans as an active component}

Traditionally in information fusion, one has tried to exploit human capabilities in designing interfaces and architectures of systems. For instance, the JDL model level 5 identified areas which needed more focus (such as techniques to direct attention). However, the interaction with an information fusion system has not been considered in a wider perspective, i.e. the information fusion system typically has not been put into the context of an information fusion process (as illustrated in figure 1 ), an information fusion process may include one or more users, one (or more) information fusion systems, also, the users usually belong to some sort of organisation and environment. Hence, the information fusion system, as such, does not define the information fusion processes.

To be able to capture the actual information fusion processes, one needs to consider a vast array of aspects and, most importantly, one should remember that humans have an active role. The fact that humans should have an active role has also been recognised by Blasch and Plano [11] who "see the human as an active component in the fusion process to optimize sensors to best locate and ID targets” (p. 270). However, most often, humans are viewed as mere receivers of an information fusion system's output, i.e. the receiver of fused information.

Distributed cognition can give us an appropriate approach to study humans as an actual integral part of the information fusion process (see also figure 1). In distributed cognition humans are considered as active components and the cognitive process is created in the interaction with the information fusion system and its surroundings. Distributed cognition is about creating something more which cannot be achieved through the individual parts, i.e. distributed cognition considers individuals with different roles and tasks and the overlap between them, as a result, a new shared understanding and meaning emerge. Indeed, “... effective and efficient interactions between the fusion system and the user, the sum (as defined in the metrics) should be greater than the separate parts" [32] (p. 466). Hence, the user is no longer only viewed as a mere observer of an information fusion system; rather, the humans are included in the information fusion process.

For example, the human user can play an important and active role for the overall information fusion process as a feedback provider. The user can gives responses to the system and the system can continue to process that information. In fact, it has been shown that human operators can be an active part of sensor networks as an information source [24]. Actually, it has also been argued that "without human inputs, the DFS [Data Fusion System] refinements, based only on the data received, may be time consuming and useless" [32] (p. 463). It can be argued that through a distributed cognition perspective one could more easily identify and, thus, enhance the interaction between the human and the fusion system and therefore, enable us to rethink the user's role in information fusion. 


\subsection{Tackling problems outside-in}

With a distributed cognition perspective one would tackle problems/situations from the outside in, beginning with the social and material settings, that way, culture, organisation, context, and history can be introduced in the information fusion process.

Specifically, a distributed cognition perspective would enable the organisational perspective to be incorporated into the information fusion process. The significance of the organisation should not be underestimated since norms and goals of an organisation can directly guide the user, the decision maker; consequently, the organisation affects the usage of information fusion systems. Moreover, it can be suggested that it is important to consider an organisational perspective rather than only a group/team perspective [25] since we, in information fusion, often deal with people who have the authorization and the responsibility to make a decision in a certain area. In fact, it has been acknowledged that the traditional model in the command and control process, the well known OODA loop, is relatively static and does not explicitly take the surrounding environment into account. To address some of those problems, the DOODA loop has been developed [12]. In fact there is a strong correspondence between the concepts of sensemaking $[12,17]$ in the DOODA loop and concepts of distributed cognition. In our opinion, there is a possibility to extend the perspective on information fusion by introducing terminology and concepts from distributed cognition.

Furthermore, as previously mentioned, looking at problems from the outside in would enable history to be included to the information fusion process. This is, to some extent, already utilised in techniques such as "tracks/path" where, for instance, the history (past) is used to predict the future. Also, Blasch and Plano [30] point out "the user maintains a cognitive model of the things that are going on and updates the cognitive model based on the context. Context includes the task of interest, the priority information, and some history of what the user has experienced in the past” (p. 198). The significance of the past to the information fusion process should not be compromised and having a distributed cognition perspective could explicitly account for that. In fact, one could argue that information fusion is all about fusing the past, present and future.

One also needs to consider the specific user's interaction with the information fusion system, e.g. human factors. For instance, Bisantz at al. [23] identified important factors which influence the usage of an information fusion system, and, thus, the information fusion process. For instance, situation awareness is a very important factor in order to achieve a common understanding between the different individuals.

One important aspect of situation awareness, which also plays an important role for distributed cognition, is to which extent a task can be automated in a computer system without reducing human situation awareness. In other words, when a system is automated it might perform operations which the user is unaware off, hence, the user's situation awareness decreases. Nevertheless, automating the information fusion process is still foremost a research issue [26]. Many researchers have considered the technical aspects, however, the users of the system are the ones making the decisions, and therefore, they are an essential part when automating the information fusion process.

The balance between the organisation, the individuals and the automation of the information fusion system is crucial; as a consequence, one needs to consider many different aspects, including trust, in order to achieve an overall effective information fusion process. The distributed cognition framework could be used to facilitate this balance. We argue that there is a need to rethinking the concept of "the JDL model level 5" and acknowledge the human and the surrounding environment as parts of the information fusion process. This is also in the line with Blasch and Plano [30] who argue that context determines user refinements, i.e. the users' action/activity. Distributed cognition, in our opinion, can provide an additional value, by providing an approach to achieve this incorporation and capture information fusion processes.

\subsection{A natural shift in information fusion}

It can be argued that the proposed development of information fusion research (i.e. the usage of distributed cognition) follows recent developments in cognitive science. Traditionally, in cognitive science one has only been interested of the inside of individuals (e.g. memory), however, in recent years the interest has been broadened to also consider cognitive science as something which emerge in the interaction with other individuals or environment i.e. distributed cognition. Today, distributed cognition is a commonly used and accepted perspective within cognitive science. The shifts from individual interaction to more distributed/collaborative interactions are also notable when examining the recent development of decision support technology [27].

In information fusion, researchers have begun to recognise the distributed nature that, in fact, is present in a wide range of information fusion processes [13, 22-23]. For instance, researchers have acknowledged the need to address the distributed nature of information fusion by referring to the distributedness of architectural issues and algorithmically issues (referred as "Distributed Data Fusion", DDF) [13]. This is an important starting point which establishes the recognised distributed nature of information fusion, however, we propose that information fusion needs to be further developed and also take the human aspect into account. Distributed cognition can, as is proposed to HCI [19], be a new foundation for information fusion. This would be a natural development because one needs to consider the environment in which an information system operates to capture the actual information fusion process and hence, enable the breaking of the previously mentioned "HCI bottleneck". 


\section{Conclusions and future work}

This paper has briefly reviewed some of cognitive science's potential contributions to information fusion, in particular the JDL model, and suggested that adopting a distributed cognition perspective can be a valuable complement to existing approaches to understanding and modelling information fusion, in particular information fusion processes. There is much technological research on how to create effective information fusion systems, however, it is suggested that one also needs to consider distributed cognition to create an effective information fusion process. One can argue that there is a difference between thinking of the information fusion process as a process in the information fusion system versus the information fusion process, as defined herein. One need go beyond the HCI and take the insights from cognitive science (e.g. distributed cognition) into account in order to capture the actual information fusion process.

However, there is still a question of integrating the different theories in cognitive science, including distributed cognition, into information fusion, in order to capture the actual information fusion process. In particular, distributed cognition can provide crucial elements of a theoretical framework and an analytical methodology which could be used to capture the nature of information fusion processes and their embedding in the support of organisational, individual and automatic decision processes. This would be difficult to achieve with the more traditional static approaches in cognitive science such as cognitive task analysis [33]. Above all, the advances in cognitive science have yet to be incorporated in the information fusion process and this provides a great challenge and will be further examined in future studies.

\section{Acknowledgements}

This work was supported by the Information Fusion Research Program (www.infofusion.se) at the University of Skövde, Sweden, in partnership with the Swedish Knowledge Foundation under grant 2003/0104.

\section{References}

[1] E. Waltz and J. Llinas. Multisensor Data Fusion. Artech House Inc., Northwood, Ma, 1990

[2] L. J. De Vin., A. H. C Ng., J. Oscarsson and S. F Andler. Information fusion for Simulation Based decision support. Proc. of Flexible Automation and Intelligent Manufacturing (FAIM2005), Spain. 2005.

[3] A. Ross and A. Jain. Information fusion in biometrics. Pattern Recognition Letters 24: 2115-2125, 2003

[4] B. V. Dasarathy. Information Fusion- what, where, why, when and how? Information Fusion. 2: 75-76. 2001

[5] Hall and A. N Steinberg. Dirty secrets in multisensor data fusion. In: D.L. Hall J. Llinas (edt), Handbook of multisensor data fusion. CRC Press Inc., Florida USA. 2001.

[6] G. Jakobson, L. Lewis and J. Buford. An approach to integrated cognitive fusion. Proc. $7^{\text {th }}$ Int. Information Fusion Conf. Stockholm, Sweden 2004.

[7] G. Looney and L. R. Liang. Cognitive situation and threat assessments of ground battlespaces. Information Fusion 4: 297-308. 2003.

[8] D. L. Hall and A. McMullen. Level 5: Cognitive Refinements and Human-Computer Interaction. In: Mathematical techniques in Multisensor Data Fusion. Artech House, Inc. US. 2004

[9] M. M. Kokar., M, D. Bedworth and C. B. Frankel. Reference model for data fusion systems. Proc. Sensor Fusion: Architectures, Algorithms, and Applications IV. SPIE Vol. 4051, p. 191-202, 2000

[10]B. V. Dasarathy. Information Fusion in the context of human-machine interfaces. Information Fusion. 117-118. 2005

[11]E. Blasch and S. Plano. JDL Level 5 fusion model: user refinements issues and application in group tracking. SPIE vol 4729, Aerosense. 270279, 2002.

[12]B. Brehmer. The dynamic OODA loop: amalgamating Boyd's OODA loop and the Cybernetic approach to command and control. Proc. of $10^{\text {th }}$ international command and control research and technology symposium. Virginia, June 2005

[13] J. Llinas., C. Bowman., G. Rogova., A. Steinberg, E. Waltz and F. White. Revisiting the JDL Data Fusion Model II. Proc. $7^{\text {th }}$ Int. Information Fusion conf. Stockholm, Sweden, 2004.

[14] M. J. M Hall, C. S A. Hall \& T. Tate, Removing the HCI Bottleneck: How the Human Computer Interaction (HCI) affects the performance of Data Fusion System. In: Hall D. L \& Llinas J (ed.), Handbook of Multisensor data fusion, CRC Press, Florida USA. 2001

[15]M. Bedworth and J. O’Brien. The omnibus model: A new model for data fusion. Aerospace and Electronics Systems Magazine, 15(4):30-36, 2000.

[16] J. Boyd. A discourse on winning and loosing. Maxwell Air Force Base, Al: Air University Library document No. M-U 43947. 1987

[17] J. T. Nosek. Social construction of knowledge in teams: Issues for computer support. In: $\mathrm{M}$. McNeese, E. Salas and M. Endsley (edt.), New trends in cooperative activities: understanding system dynamics in complex systems, Human factor and ergonomic society: US. 2001.

[18]E. Hutchins. Cognition in the wild. MIT Press, Cambridge.1995.

[19] J. Holland, E. Hutchin, D. Kirsh. Distributed Cognition: Towards a new foundation for Human -Computer Interaction research. ACM Transactions on Computer- Human Interaction, 7:2, 174-196, 2000 
[20]S. Schrire. Interaction and cognition in asynchronous computer conferencing. Instructional science 32:475-502, 2004.

[21] J. S. Busby. Error and distributed cognition in design. Design studies. 22: 3 233-254. 2001

[22]S. Snell. The dissemination and fusion of geographical data to provide distributed decision making in a network-centric environment. The $9^{\text {th }}$ international command and control research and technology symposium. Washington DC, USA 2004

[23] A. M. Bisantz, J. Llinas and C. G. Drury. Issues in Aided Adversarial Decision-Making performance. In: M. McNeese, E. Salas and M. Endsley (edt), New trends in cooperative activities: understanding system dynamics in complex systems. Human factor and ergonomic society: US. 2001.

[24] T. Kaupp, A. Makarenko., S. Kumar, B Upcroft, S. Williams. Operators as Information Sources in Sensor Networks. IEEE/RSJ International Conference on Intelligent Robots and Systems, Edmonton, Canada, 2005

[25] G. M. Markas. Decision support systems in the 21st century. Prentice hall: New Jersey, US. 1999.

[26] S. Ahlberg., P. Hörling., K. Jored., C Mårtenson., G. Neider, J. Schubert., H. Sidenbladh., P. Svenson., P. Svensson, K. Undén and J. Walter. The IFD03 Information Fusion demonstrator. Proc. of 7th Int. Information fusion Conf. Stockholm, Sweden 2004

[27] J. P. Slim, M. Warkentin., J. F. Courtney., D. J. Power., R. Shada., C. Carlsson. Past, Present, future of decision support technology. Decision Support system. Vol. 33, 11-126, 2002.

[28] A. Steinberg. An approach to threat assessment. Proc. of $8^{\text {th }}$ Int. conference of Information fusion. 2005

[29] E. Blasch and S. Plano. Level 5: User refinements to aid the Fusion Process. Proc. Multisensor, Multisource, Information Fusion: Architectures, Algorithms, and Applications. SPIE Vol. 5099. 2003

[30]E. Blansch and S. Plano. Cognitive Fusion Analysis Based on Context. Proc. Multisensor, Multisource, Information Fusion: Architectures, Algorithms, and Applications. SPIE Vol. 5434. 2004

[31] Y. Rogers. Distributed Cognition and Communication. (in press) Encyclopedia of Language and Linguistics ( ${ }^{\text {nd }}$ Ed.). 2006

[32] E. Blasch. Situation, Impact, and User refinement. Proc. Processing, Sensor Fusion, and Target Recognition XII. SPIE Vol. 5096, 463-472. 2003.

[33] Y, Rogers. A Brief introduction to Distributed Cognition $@$. 1997. Available at: http://www.slis.indiana.edu/faculty/yrogers/publi cations.html 